\title{
Depletion in a distributed Brillouin fiber sensor: practical limitation and strategy to avoid it
}

\author{
Luc Thévenaz ${ }^{*}$, Stella Foaleng Mafang and Jie Lin ${ }^{\mathrm{a}}$ \\ EPFL Swiss Federal Institute of Technology, Group for Fibre Optics, Institute of Electrical Engineering, \\ STI IEL GR-SCI Station11, CH-1015 Lausanne, Switzerland \\ ${ }^{a}$ Permanent address: Bucknell University, Dept. of Electrical Engineering, Lewisburg PA 17837, USA
}

\begin{abstract}
Energy transfer between the interacting waves in a distributed Brillouin sensor can result in a distorted measurement of the local Brillouin gain spectrum, leading to systematic error. We demonstrate here that this behavior can be fully and precisely modeled, and an excellent quantitative agreement is found with experimental tests. Strict guidelines can be enunciated from this description to make the impact of depletion negligible, for any type and any length of fiber.
\end{abstract}

Keywords: Fiber optics, Optical fiber sensors, distributed fiber sensor, stimulated Brillouin scattering, Nonlinear optics.

\section{INTRODUCTION}

The detrimental impact of depletion was soon identified as a potential source for systematic errors in a distributed fiber sensor based on stimulated Brillouin scattering (BOTDA $)^{1,2}$. As a result of the cumulated energy transfer between the $\mathrm{CW}$ probe wave and the pump pulse due to the stimulated Brillouin interaction, the amplitude of this pulse turns out to be modified and can lead to a pump power that is frequency-dependent once the cumulated energy transfer is substantial, i.e. predominantly at the far end of the sensing fiber. This effect is of more importance when the Brillouin spectrum is uniform along the fiber, since the cumulated gain is maximized when the frequency difference between pump and probe exactly matches the Brillouin shift. This effect can be clearly shown in Fig.1, where the pump power at the output of a uniform fiber is measured as a function of the pump-probe frequency difference. The depletion is stronger when the probe power is larger, since more power is required from the pump to deliver the same gain.

This frequency-dependent pump power will provide a biased measurement of the gain spectral distribution if the pump pulse enters a section where the Brillouin gain central frequency is shifted with respect to the gain spectrum in the long

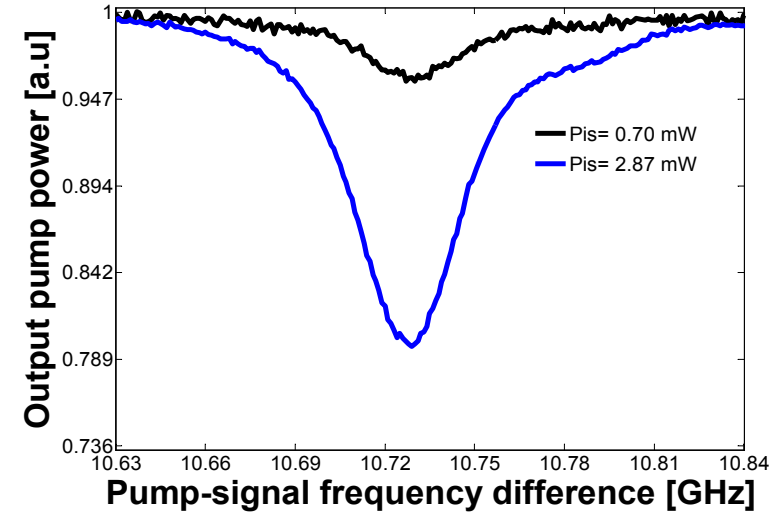

Fig.1: Pump peak power as function of the frequency difference of the pump and the signal for two different values of the CW probe power $P_{i s}$, measured at the output of a $1 \mathrm{~km}$ uniform fiber, showing the severe depletion effect experienced by the pump. uniform preceding section. Very recently, we have fully modeled this effect, demonstrating that the systematic error on the determination of the Brillouin shift is maximum when the central frequencies between the long uniform preceding section and the measured segment are separated by a frequency equal to $\sim 1 / 4$ of the FWHM of the Brillouin gain spectrum (a separation of about $7-10 \mathrm{MHz}$ in normal conditions).

\section{EXPERIMENTAL MODEL VALIDATION}

These conditions could be exactly realized experimentally, by locally heating a $10 \mathrm{~m}$ segment at the distant end of a $1 \mathrm{~km}$ uniform standard fiber. The local gain spectrum measured at the center of the heated segment using a classical BOTDA technique is shown in Fig. 2 for increasing $\mathrm{CW}$ probe power. As described in our recent paper ${ }^{3}$, it can be observed that the measured spectrum is skewed, leading to a systematic error in the determination of the central frequency.

\footnotetext{
*luc.thevenaz@epfl.ch; phone +41 21693 4774; http://people.epfl.ch/luc.thevenaz
} 

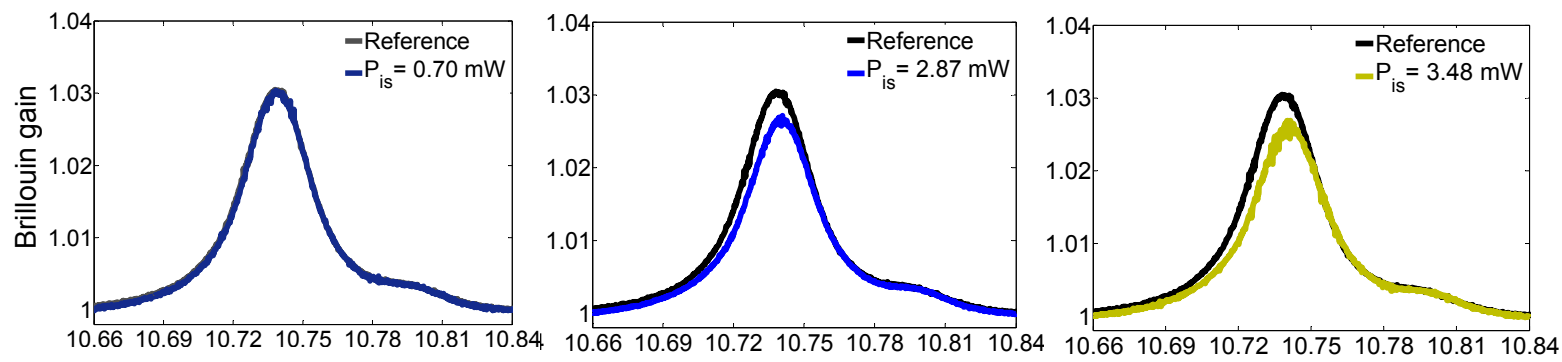

pump-signal frequency difference $[\mathrm{GHz}]$

Fig.2: Brillouin gain spectrum measured within a $10 \mathrm{~m}$ segment of fiber, placed at the end of a long $1 \mathrm{~km}$ section of uniform fiber and shifted by 10 degrees in temperature. The gain spectrum is measured for different powers of the $\mathrm{CW}$ probe signal and for a fix pump pulse peak power of $69 \mathrm{~mW}$. For comparison a reference spectrum is shown in gray, measured without propagation in the long uniform section, so with a total absence of depletion. The actual measured gain spectrum shows a distorted skew distribution with a slightly up-shifted peak position

The effect is even more striking when the following simple comparative test is carried out: the distributed measurement of the Brillouin shift is performed successively when the short segment is placed at the near end and at the far end of the fiber. This can be simply realized without modifying the experimental layout by swapping the connections of the sensing fiber to the instrument, so that the pump pulse sees alternatively the short segment before and after propagation in the long uniform section. In the first case, the pump will show no depletion while in the measured segment, since it enters it immediately, without experiencing prior interaction, and this measurement can be used as a reference and as a proof of the absence of any other biasing effect. In the second case, the pump will be fully subject to depletion and an error must be observed in the determination of the Brillouin shift, which should depend on the amount of depletion.

Frequency-shifted segment placed near the fiber input

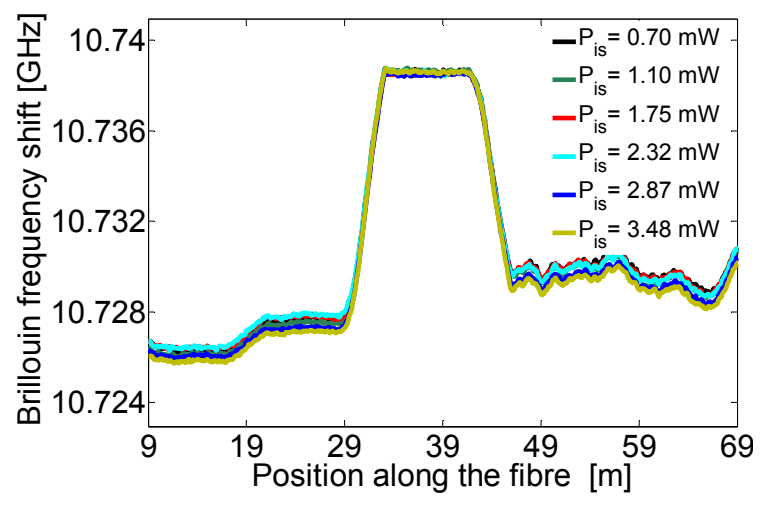

Frequency-shifted segment placed near the fiber output

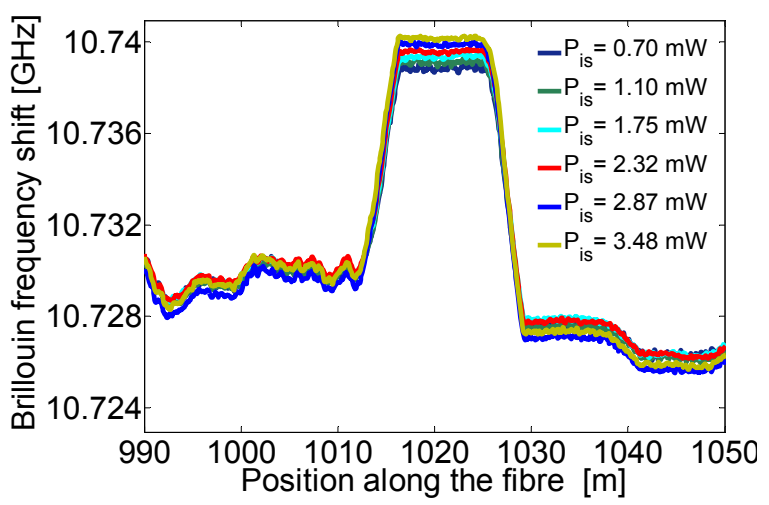

Enlarged view of the short segment

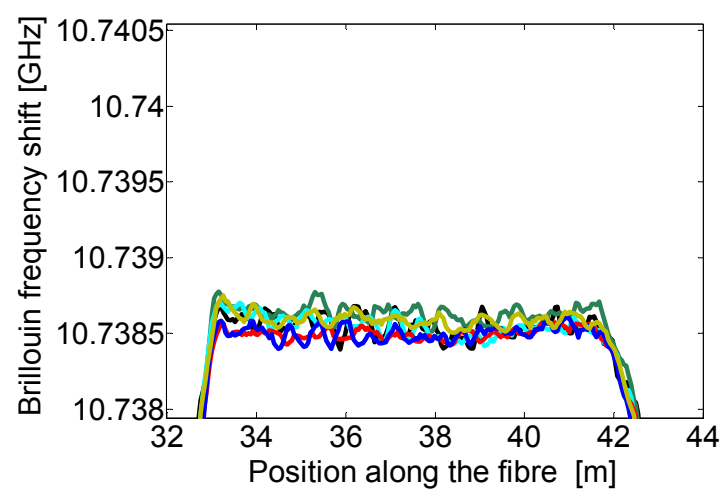

Enlarged view of the short segment

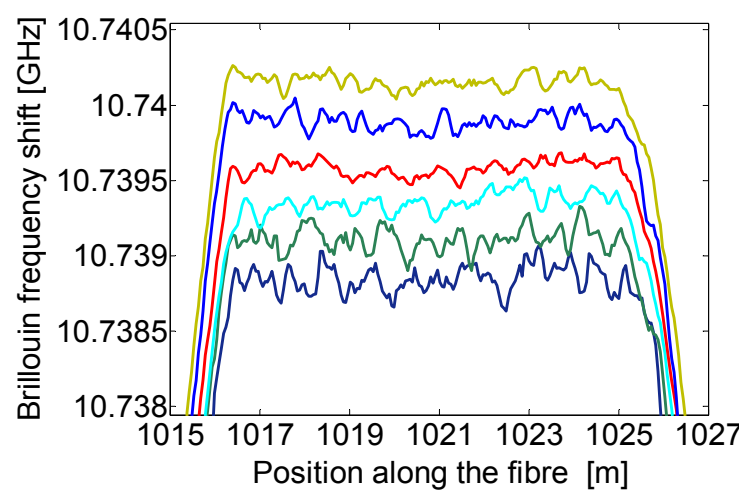

Fig.3: Brillouin frequency shift as function of the position for different powers $P_{i s}$ of the $\mathrm{CW}$ signal, in the vicinity of a $10 \mathrm{~m}$ segment of fiber, placed at one end of a long $1 \mathrm{~km}$ section of uniform fiber and shifted by 10 degrees in temperature. Top row: when the frequency-shifted segment is placed near the fiber input. Bottom row: when the same short segment is placed near the fiber output. Experimental layout and power settings are identical to Fig. 2. 
Fig. 3 illustrates perfectly this effect, using an experimental configuration identical to Fig. 2. It can be clearly seen that the value of the Brillouin shift depends on the power of the $\mathrm{CW}$ probe wave, which scales the amount of depletion at constant pump power. It should be kept in mind that this testing configuration (long uniform segment followed by a slightly frequency-shifted measurement zone) is the worst case situation and is very unlikely to occur in the reality in this exact configuration. It therefore constitutes a very reliable test of the robustness of a configuration to the effect of depletion.

The model $^{3}$ also predicts the amount of depletion $d-$ representing the fractional change of the pump pulse power resulting from the energy transfer between pump and probe that is tolerable to avoid exceeding a given error in the worst case situation. This could be tested using the above described configuration and the results are shown in Fig. 4. The agreement between model and experimental data is excellent and confirms that a $1 \mathrm{MHz}$ max. error on the Brillouin shift determination tolerates a $17 \%(0.8 \mathrm{~dB})$ change on the pump power through depletion.

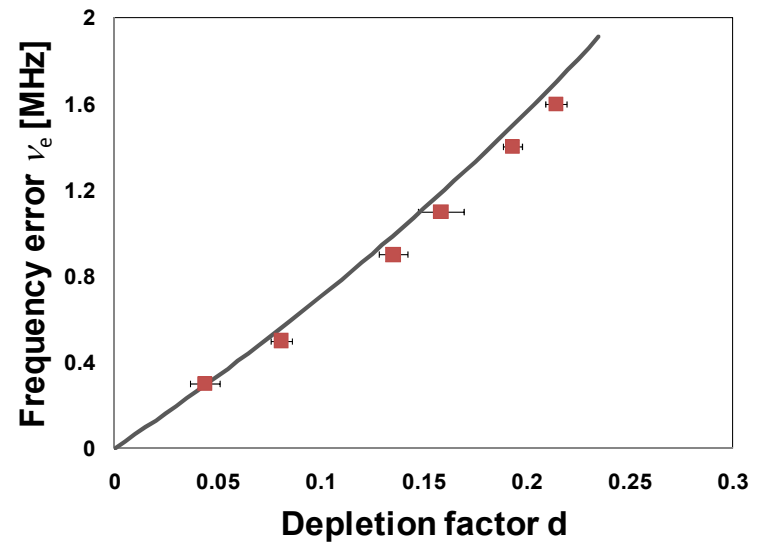

Fig.4: Measured frequency error as a function of the measured pump depletion, in the worst case situation of a fiber segment showing a Brillouin frequency shifted by $10 \mathrm{MHz}$ after a long uniform fiber section (same configuration as in Fig. 2-3). The solid line shows the analytical model prediction.

\section{LIMIT TO SIGNAL POWER TO KEEP DEPLETION TOLERABLE}

Under the assumption of a small gain experienced by the $\mathrm{CW}$ probe wave - that is reasonable in a high spatial resolution and long range sensor where the probe gain is normally in the percent range - our model ${ }^{3}$ demonstrates through a very simple expression that the amount of depletion experienced by the pump pulse only depends on the $\mathrm{CW}$ input probe power $P_{i s}$ for a given Brillouin gain $g_{o} / A_{\text {eff }}$ and nonlinear effective length $L_{e f f}$. We straightforwardly extend here this expression to the case shown in Fig. 5, where $2 \mathrm{CW}$ signal waves can be simultaneously present and are placed at the same frequency distance above and below the pump pulse signal:

$$
1-d=\mathrm{e}^{-\frac{g_{B}}{A_{e f}}\left(P_{i S L}-P_{i S U}\right) L_{e f f}}
$$

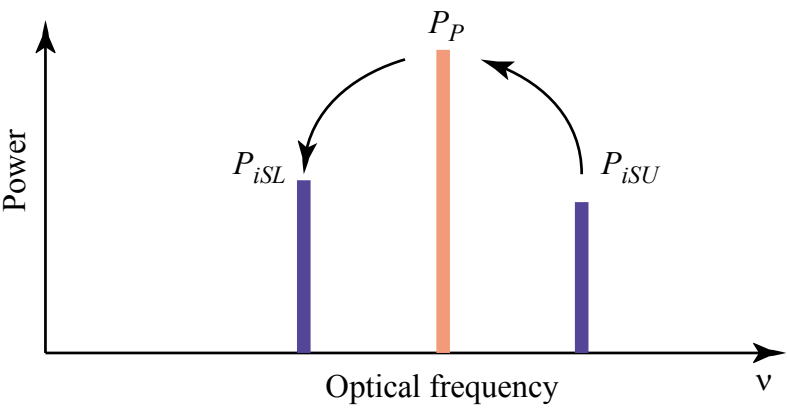

Fig.5: General spectral arrangement of pump and probe signals, considering most practical situations in real Brillouin distributed sensors. The arrows indicate the directions of the power transfer due to the interaction. The sensors may operate in gain regime $\left(P_{i s L}=0\right)$, in loss regime $\left(P_{i s L}=0\right)$ or in double-sideband configuration $\left(P_{i s L}=P_{i s U}\right)$.

This expression is helpful to indifferently describe a sensor operating in the gain regime $\left(P_{i s U}=0\right)$ or in the loss regime $\left(P_{i s L}=0\right)$, or even in the simultaneous presence of $2 \mathrm{CW}$ waves, equally spectrally-shifted from the pump, as conveniently generated using the modulation sidebands of a modulator operating in a suppressed-carrier configuration ${ }^{4}$.

This expression was also challenged by experimental tests, in the case of a sensor operating in gain regime $\left(P_{i s L}=0\right)$. The results are shown in Fig. 6, together with the prediction of the model. The agreement is again excellent and the fact that the amount of depletion is independent of the pump pulse power is experimentally proved in a first order approximation (small gain regime).

Since the model provides an expression giving the tolerable depletion $d$ that will secure an error on the frequency which does not exceed a given limit in the worst case situation, the maximum CW signal power can be easily determined by reversing Equ.1:

$$
P_{i s L}-P_{i s U}<-\ln (1-d) \frac{A_{\text {eff }}}{g_{B} L_{e f f}} \stackrel{L \rightarrow \infty}{=}-\ln (1-d) \frac{A_{e f f}}{g_{B}} \alpha
$$

This expression clearly shows that configuring the sensor to propagate a $\mathrm{CW}$ probe signal made of 2 modulation sidebands of equal amplitude can render the system independent of the $\mathrm{CW}$ signal power and thus very robust to depletion. This was soon identified as a clear asset of the double sideband configuration ${ }^{4-6}$ and it is here fully proved by the modeling. In that case, one of the sideband must be filtered out just before detection, since the Brillouin response is opposite of equal amplitude on each sideband. Actually this full compensation of the depletion by the spectrally 

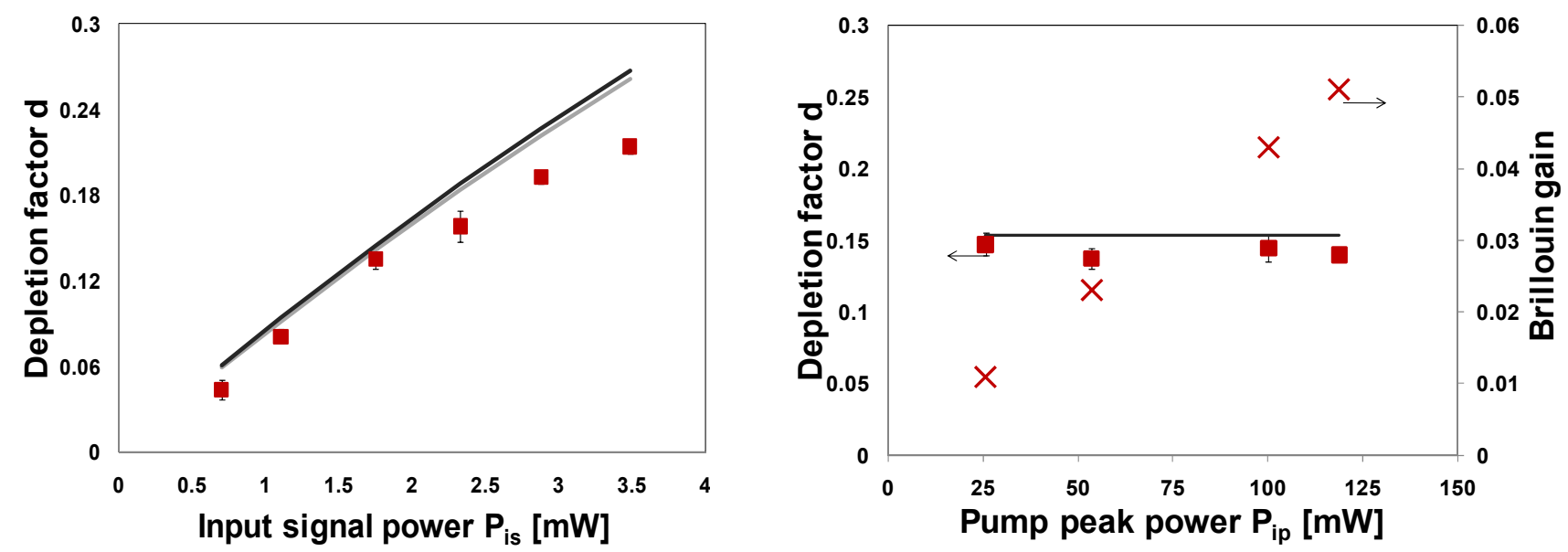

Fig.3: Measured depletion factor $d$ as a function of the interacting wave powers, for the same experimental configuration as in Fig. 2-4. The solid lines show the predictions from the model.

Left: the dependence of the amount of depletion experienced by the pump pulse on the CW probe signal power is in very good agreement with the model prediction. The fact that the real depletion is a bit inferior is probably due to the imperfect Brillouin spectral uniformity along the fiber.

Right: while the pump pulse power scales the amount of gain, it has no impact on the amount of depletion, as predicted by the model (here the signal power is kept constant $P_{i s}=1.9 \mathrm{~mW}$ )

symmetric sidebands is correct in a first order approximation, in which the amplitude change of the probe waves is considered proportionally negligible. We have carried out a $2^{\text {nd }}$ order study, to be presented in a further publication, that demonstrates a slight dependence on the probe power, which is 100 times smaller than in a single sideband configuration (gain or loss regimes). With such a tolerance the probe power can reach several milliwatts before the depletion starts to be significant. In this situation the system is very likely limited by the onset of amplified spontaneous Brillouin emission.

These experimental results, fully consistent with the modeling ${ }^{3}$, lead to the following guidelines to design a depletionrobust sensor:

1. Since the absolute sensor response is given by $\Delta P_{s}=\left(g_{o} / A_{\text {eff }}\right)\left(T / V_{g}\right) P_{P} P_{S}$ (T: pump pulse width) and the depletion is independent of the pump pulse power $P_{P}$ to first order and highly dependent on the CW probe power $P_{S}$, this latter must be kept as low as possible while the power of the pulse pump $P_{P}$ can be freely increased. This disqualifies all configurations based on an intense $\mathrm{CW}$ wave and a weak pulse.

2. The robustness to depletion is massively improved by using a double-sideband configuration, in which no practical limit is observed before the onset of amplified spontaneous Brillouin emission.

These conclusions show that the depletion can be fully controlled and made negligible by a proper sensor design, without other actions on the sensing fiber.

\section{REFERENCES}

1. T. Horiguchi, K. Shimizu, T. Kurashima, M. Tateda and Y. Koyamada, "Development of a distributed sensing technique using Brillouin scattering," Journal of Lightwave Technology 13(7), 1296-1302 (1995)

2. E. Geinitz, S. Jetschke, U. Röpke, S. Schröter, R. Willsch and H. Bartelt, "The influence of pulse amplification on distributed fibre-optic Brillouin sensing and a method to compensate for systematic errors," Measurement Science and Technology 10(2), 112 (1999)

3. L. Thevenaz, S. Foaleng Mafang and J. Lin, "Impact of pump depletion on the determination of the Brillouin gain frequency in distributed fiber sensors," in 21st International Conference on Optical Fiber Sensors, pp. Paper 77537210, SPIE, Ottawa, Canada (2011).

4. M. Niklès, L. Thévenaz and P. A. Robert, "Simple distributed fiber sensor based on Brillouin gain spectrum analysis," Opt. Lett. 21(10), 758-760 (1996)

5. S. Diaz, S. Mafang-Foaleng, M. Lopez-Amo and L. Thevenaz, "A High-Performance Optical Time-Domain Brillouin Distributed Fiber Sensor," IEEE Sensors Journal 8(7), 1268-1272 (2008)

6. A. Minardo, R. Bernini and L. Zeni, "A Simple Technique for Reducing Pump Depletion in Long-Range Distributed Brillouin Fiber Sensors," Sensors Journal, IEEE 9(6), 633-634 (2009) 